\title{
An Investigation into the State of Status Planning of Tiv Language of Central Nigeria
}

\author{
Atonde Terkimbi \\ Department of English Language and Applied Linguistic \\ University of Birmingham, United Kingdom \\ 664 Bristol Road, Selly Oak, Birmingham, B29 6BJ, United Kingdom \\ E-mail: tfa250@bham.ac.uk /atondeterkimbi@gmail.com
}

Doi:10.7575/aiac.alls.v.7n.2p.129

URL: http://dx.doi.org/10.7575/aiac.alls.v.7n.2p.129
Received: 19/11/2015

Accepted: 21/01/2016

\begin{abstract}
The Tiv language is one of the major languages spoken in central Nigeria. The language is of the Benue-Congo subclass of the Bantu parent family. It has over four million speakers spoken in five states of Nigeria. The language like many other Nigerian languages is in dire need of language planning efforts and strategies. Some previous efforts were made to plan the language and government too has made policies to back up such efforts. This study therefore examines empirically the current state of the status planning of the language and its implication for the future and survival of the language. Status planning deals with the allocation of functions to a community's language(s). The functions investigated here include the educational, the media and home functions and it has been discovered that the language has a weak functional status. This therefore requires some conscious and deliberate language engineering strategies to be set in motion to rescue the language from its present bleak and weak functional status, to which end some recommendations have been made.
\end{abstract}

Keywords: Language planning, Status planning, Nigerian languages, Tiv language

\section{Introduction}

Nigeria is a multimodal multilingual society (if we follow Fishman's (1972) classification of nations as either amodal, unimodal or multimodal) with several great linguistic, cultural, religious and political traditions. While Crozier, Blench and Hansford (1992) keep the number at over 250, Ethnologue (2015) lists 527 languages with varying degrees of endangerment. These figures present a picture of what the linguistic composition looks like even if some seem exaggerated.

Tiv language is one of the major languages spoken in central Nigeria in the states of Benue, Taraba, Nasarawa, Plateau and some parts of Cross River with a numerical strength of over 4 million speakers. It is the major language spoken by the Tiv people who occupy the greater part of the Benue Valley. The language co-exists with other languages like Idoma, Igede, Jukun, Etulo, Abakwa and Alago. Considering the fact that this language is a major language in central Nigeria and it coexists among other languages, the need to consciously plan it to make it more viable and usable within its community becomes very expedient. This is so because in multimodal speech communities issues of language planning are not a given and are not allowed to follow a natural selection process but are given attention and efforts made to ensure that all the language resources of the community are properly harnessed and utilised in such a manner that does not disadvantage other languages or groups.

This study focuses on investigating empirically the state of the 'status planning' of Tiv language. Status planning deals with the allocation of functions to a language or among a community's languages (Cooper 1989) to enable it/them serve the purposes for which they are supposed. The primary concern of status planning therefore is the conscious allocation of functions to a language(s) in a speech community. This research therefore investigates the current level of planning of Tiv language in relation to the functions allocated to it or those it is serving at the moment and the implications of such status on the future of the language.

In doing so, the study seeks to provide answers to the following questions:

What are the functions the Tiv language is serving in Benue State?

Is Tiv language used in the educational system?

Is Tiv language used in the media?

Is Tiv language used in the homes?

The questions above relate to the key functions a language is expected to serve especially at the regional or national level. 
This paper is structured in seven subsections as follows: brief history of Tiv Language, theoretical framework, literature review, methodology, analysis, conclusion, recommendations and suggestions for further study.

\section{A Brief Background of the Origin and Development of Tiv Language}

The Word "Tiv" refers to the people as well as their language. The paradigm also extends to cover the ancestor of the people. Hembe (2005) affirms this that "the word 'Tiv' refers to the people, their language as well as their progenitor" (30). When used as the language it is usually prefixed with the prefix 'zwa' (Zwa-Tiv - Tiv language) as it is typical of the Bantu languages.

The Tiv Language belongs to the parent language family called the Bantu language family which is the dominant language family southern half of the African landmass spoken by over 157 million people (Benji 1989:991). There is enough internal (Gbor, 1986 and Abraham, 1940) and historical evidence to support this classification. Abraham for example states categorically that 'The Tiv are an originally Bantu tribe who have penetrated so far west as to become cut off from the main body and isolated among the Sudanic people' (5). Hembe (2005) comments relevantly on the authenticity of Abraham's conclusion that

on the basis of linguistic affinities, he (Abraham) along with other scholars demonstrated the close connection between the Tiv and the Bantu Nyanza. The language similarity is so striking that no one is left in doubt that Tiv is a real Bantu and not just a Semi-Bantu language. This striking linguistic similarity suggests that the Tiv and the Bantu Nyanza once lived together or at least they lived as neighbours (35).

But the most specific subclass to which the Tiv language belongs is the Benue-Congo language family. Hembe (2005) backs up the basis for this subcategorisation. According to him 'a more generally accepted suggestion, which is, also supported by empirical evidence, is that the Tiv migrated to their present location from the Congo region of Central Africa' (35).

Other scholars too agree with the fact that the Tiv were not originally of this place where they are today. Makar (1994:5) also maintains that the Tiv people migrated along the Benue coast through the Cameroon highlands as a result of increasing pressures and hostilities among their neighbours, wars and hardship, to the present location they are among the valleys of rivers Benue and Katsina-Ala. Yuhe (1978) too suggests that Tiv people faced with the hostility of their neighbours, abandoned their home and left for safety (Yuhe in Hembe 2005:35). The Tiv are located in five states of central Nigeria - Benue, Nasarawa, Taraba, Plateau and some parts of Cross River. Their principal occupation is farming as is the case with many of the languages within its family as opposed to the Afro-Asiatic and Sudanic languages whose speakers are predominantly nomads and traders.

The Tiv language during its early period of growth and development had a lot of contacts with other languages like the Bafum, the Hausa, Fulani, The Jukun, Chamba and the Kuteb. This contact was as a result of trade and migration, and these languages have had considerable influences on the development of the Tiv language. Many of the words found in Tiv language are of foreign origin and mostly of the Hausa-Fulani, Chamba and Jukun languages.

One of the major influences on the development of the Tiv language is the Dutch Reform Church Mission (DRCM) which came under the auspices of the United Sudan Mission (SUM) to Tiv land in 1911 and settled at Sai village in Shitire (Akiga 1939:1). The missionaries are acknowledged for this role because they emphasised the use of the Tiv language or other indigenous languages, as a matter of policy, in their evangelisation mission. As a result, they developed the Tiv from a purely oral to a written language. They developed the Tiv alphabet from the English alphabet with the exception of two letters "X" and "Q" from Tiv which are present in English. Gbor (1986) summarises that

The idea to develop and reduce Tiv into a written language was first enacted by the Dutch Reformed Missionaries (DRCM) who arrived in Tivland from South Africa in 1911. They adopted most of the English alphabets (sic) exception of the "Q" and "X" which are truly conspicuously absent in Tiv pronunciation. The DRCM developed the language and taught all Christian and other subjects in it (1).

They also wrote books of grammar and liturgical literature, which culminated in the translation of the Bible from English to Tiv language in 1964. The translation of the Bible from English to Tiv is a major impetus to the development of Tiv language because it has helped in preserving the language in its original form. Up till now, the Tiv Bible called "Bibilo" still remains one of the most authentic books housing the Tiv language that can be looked upon as a normative "standard".

The DRCM apart from developing and making available written literature also promoted the teaching and learning of Tiv language. They established schools and ensured the teaching, learning and use of Tiv language as medium of instruction. This contributed immensely to the development of Tiv language. The N.K.S.T Church commonly called the "Tiv Church" - by Non-Tivs is a lexical metamorphosis of the DRCM. However, the granting of independence to the Tiv Church from its parent mission in 1957 witnessed a decline of interest in Tiv language. The evangelists who were assigned the duty of education in mission schools initially took Tiv language seriously but with time their commitment dwindled, and as a result the language no longer had an impact. They did not continue with the vigour with which their predecessors - the DRCM and CRC - promoted Tiv Language (Gbor 1986:1). 
The arrival of other religious groups into Tiv land did not do any good to Tiv language. This is because many of them did not favour or promote the use of Tiv language in the conduct of evangelism and other liturgical rites and activities. They rather preferred English language and used interpreters to reach out to the people. This therefore slowed down the pace of the growth of Tiv language in many parts of Tiv land - where the DRCM were not in control of missionary activities.

The post-independence political leadership too did not pay any particular attention to indigenous languages. Though indigenous languages have been given considerations in the educational policy, but Government has failed to implement its policy. The teaching of the Tiv language is still in effect in schools but not very effective and the teaching is not even enforced.

However, the bleak picture painted above does not completely indicate that the Tiv language has remained stagnant. Some considerable efforts have been made especially by Tiv writers in areas of grammar and literature. Some of such works include Orkar's grammar books - over ten of them written by Kpamor Orkar, Lydia Ingbiankyaa's (course books) Hen Zwa Tiv 1 - 5, Tar Homon u Botwev by G.T. Humbe (1962), Adan-Wade Kohol Ga, (1982) by Suemo Chia, Dikishonali I Tiv by Sefan Gyangyan (2000) - and few others. This is a good signal but the picture painted by the amount of literature in the language shows that there is no enough available written literature in Tiv.

This historical survey therefore shows that Tiv language has in recent times not received enough attention as compared to its early periods of development. Though this history appears in gloomy colours, the fact remains that Tiv language has a lot of prospects for development than extinction, if significant efforts are made.

\section{Theoretical Framework}

Theoretical perspectives in language planning dwell most specifically on the foci of planning, and sociolinguists, notably Joshua Fishman (1968, 1972, 1974), Charles Ferguson (1968), Einar Haugen (1971, 1983), Joan Rubin (1971), Heinz Kloss (1969) and Robert Cooper (1989) among others, have given considerable attention to these theoretical perspectives. The theoretical framework for this research is based on the foci of language planning identified by Heinz Kloss (1969) and Robert Cooper (1989). Kloss identifies two of the foci: status and corpus planning, and Cooper identifies the third: Acquisition planning. This research is modelled or based on the status, corpus and acquisition planning dimensions.

Corpus planning, according to Cooper (1989) 'refers to activities such as coining of new terms, reforming spelling, and adopting a new script. It refers in short, to creation of new forms, the modification of old ones, or the selection from alternative forms a spoken or written code (31). He enumerates the processes and concepts involved in corpus planning, namely cultivation, reform, standardization, selection, codification, modernisation and elaboration. Cooper's words clearly indicate that the focus of corpus planning is on the structure or form of the language - the corpus. That is, its spoken and written forms.

Status planning, on the other hand, "refers to deliberate efforts to influence the allocation of functions among a community's languages" (Cooper1989:99). Other scholars like Rubin (1971:340) refer to it as "Language Allocation". The most widely recognised term in linguistic discourse, however, is status planning. The emphasis of this type of planning is on the functional allocation of the language(s) within a speech community. A well-known list of language functions is the one which Stewarts (1968:22) provides in his discussion of national multilingualism. He states the following functions as the foci of status planning: Official, provincial, wider communication, international, capital, group, educational, school subjects, literary, religious, mass media and work.

The third type of language planning strategy is Acquisition Planning. While Corpus and Status planning take care of the structural and functional levels of language, Acquisition planning focuses on language teaching and learning. When the form and function of language are planned, there is usually the need for the teaching and learning (acquisition) too to be planned. Cooper (1989), its main proponent, defines Acquisition planning as "organized efforts to promote the learning of a language" (3).

As stated earlier, the present study draws on these three dimensions of language planning but focuses specifically on status planning as it relates more directly to its primary concern.

\section{Literature Review}

\subsection{The Concept of Language Planning}

The literature on language planning has several concepts, some of which include: Language Planning, Language Engineering, Linguistic Engineering, Language Development, Language Management, Language Regulation and Language Treatment. These concepts are in most cases products of lexical innovations, or preferences by some scholars. In some strict sense, there may be some little semantic variation among them which may still keep them within the same semantic continuum. In this study, the concept "Language Planning" is adopted.

Language Planning has been given several definitions by different scholars. According to Das Gupta, it is "a set of deliberate activities systematically designed to organize and develop the language resources of the community in an ordered schedule of time" (157). In Fishman's (1983) words "it is the organized pursuit of solutions to language problems typically at the national level" (79). Gorman (1973) on his part defines it as "coordinated measures taken to select, codify and, in some cases, to elaborate orthographic, grammatical, lexical, or semantic features of a language and to disseminate the corpus agreed upon' (73). 
Cooper who has had an enduring impact on language planning defines it as "deliberate efforts to influence the behaviour of others with respect to the acquisition, structure and functional allocation of their codes" (1989:45). Cooper's definition seem to have a wider scope as it embraces the three major foci of planning and also emphasises the key component: 'behaviour' and mentions 'codes' as language planning incorporates a broader semiotic scope than just language in the strict sense. This is so because language planning is intimately connected with and influenced by sociodemographic, linguistic, socio-psychological, political and religious factors (Appel and Muysken 1990:56).

\subsection{Processes and Programmes in Language Planning}

Language planning is not a random or an accidental activity but a well-conceived and executed one. It involves "the design and implementation of strategies (conscious and deliberate steps) towards the rehabilitation and optimal utilization of individual languages" (HBC Capo 1990:1). It is an activity that is very methodical, involving several processes, concepts, stages, actors and programmes. Linguists have identified these processes, concepts, etc to be very useful in language planning. One of such scholars who dwells on some of the concepts is HBC Capo (1990). According to him,

In the textbooks, mention is made of such concepts as codification, standardisation, modernization development, reform etc. ... Most of the time codification refers to writing, but it is more embracing than graphisation whereas the latter seems restricted to devising an alphabet or any other writing system (such as syllabary), the former includes the production of primers, spelling books, grammar books, dictionaries etc. Codification is like standardization except that the latter aims explicitly at a normalized (and prescribed) orthography and pronunciation prescriptive dictionary etc. As for modernization, it generally connotes expansion of the lexicon i.e. the creation and adoption of abstract and precise notions needed in philosophy, slaw, science and technology. The same applies to development except that this may not be restricted to vocabulary (1)

Capo's insight indicate that language planning is a continuing and dialectic process including orthography design, corpus planning, materials development, encouragement of language use at all levels to account for and communicate the experiences of the speakers as well as aspects of the human legacy called language (Capo 1990:2). It shows that language planning is an omnibus process with several tentacles. Language planning may be carried out for different goals such as language purification, language reform, language revival and language standardization (Lucas and Nercissians 1988:2). Cooper too agrees with Lucas and Nercissians that if the establishment of the Académie Française, the promotion of Hebrew in Palestine, the American feminist movement's campaign against sex bias in language, and the Ethiopian mass literacy campaign exemplify language planning, then language planning is directed towards a variety of ends and encompasses a variety of means (1989:29). He proceeds further that in language planning certain questions have to be answered. These are: who, plans what, for whom and how? Language planning as seen above is therefore a conscious and engaging activity involving several processes, factors and agents, and needs to be given the desired attention to achieve enduring and fruitful outcomes.

\subsection{Language Planning in Africa}

One of Africa's notable scholars, Ngugi wa Thiong'o has attributed the epileptic planning moves made by several planners and the state of African language to the continuous presence of foreign languages in Africa (2003:157). He decries this development and argues that African languages have great potentials for development and should be harnessed by African linguists and intellectuals. He commends the efforts of such scholars like of like Kwesi Kwaa Prah who is doing so much to advocate the use of African languages in all fields of learning, even in scientific thought. Others include Neville Alexander of South Africa who chaired the committee that came up with the new very enlightened South African policy on languages, and Kwesi Weridu, who long ago called on African philosophers to engage issues in African languages (Ngugi 2003:160). Several other examples mentioned by him include, the continued Ethiopean Scholarship in African languages (160-161).

Ngugi (2003) therefore sees a similar commitment by other African intellectuals as a solution to her language problem. $\mathrm{He}$ is optimistic that it can be done and as has been done in Tanzania where Kiswahili has now developed a massive vocabulary in all branches of learning (161). To him, all it takes is courage and hardwork. He cites the case of Gatua wa Mbugua, a graduate student at Cornell University who in 2003 presented and successfully defended his Masters dissertation on "Bio-intensive Agriculture" to the department of crop science. According to Ngugi "There is nothing unusual in this, what is new is the fact that the entire masters was in Gikuyu language... He wrote the entire thesis in Gikuyu before translating it for his teachers who, of course, had to evaluate the scientific content' (161). Gatua's effort is a bold step with its roots on hardwork and courage. Ngugi therefore calls for African intellectuals to do for their languages and cultures, what other intellectuals in history have done for theirs (164).

Another notable African linguist, Sammy Beban Chumbow (2003), also shares the same view as Ngugi and most specifically on language planning in education, science and technology. He says that linguists, psycholinguists and anthropological linguists agree however, that the use of the child's mother tongue as medium of instruction in the school system has significant advantages over the use of an exoglossic language (170). He further adds that "the effective mobilisation of the masses of the rural population for national development requires the democratisation of access not only in an exoglossic language but also in a language (or languages) the people know best: an African national language" (171). He summarises the psychological and educational advantages of using the child's mother 
tongue in the school system to include a greater participation of Africans in the life of their nation, a better development of adult education, as a means of transmitting and preserving cultural values, a greater interaction between ethnic groups and a higher degree of national awareness, a better quality of human resources for national development and a democratisation of access to scientific knowledge and technology to the benefit of the masses of the rural population (172-173). These advantages as enumerated by Chumbow really make a lot of promise of benefit to African languages and African development.

However, there are those who have some objections to the thinking of Chumbow (2003) and his contemporaries. Some of such objections claim that African languages have no grammar. Others believe that the use of indigenous languages will impede the acquisition of the official languages. Children who attend schools where African languages are used will be at a disadvantage. Chumbow (2003), like Ngugi, argues against these objections. He calls them "objectionable objections" without any strong or rational basis. He however admits that the use of African languages in Africa has some key practical problems. The first he identifies is the inadequacy of scientific and technical vocabulary. The second point he raises is the multiplicity of languages in Africa. The third dwells on cost and resources and the last is the shortage of adequately trained teachers to teach in African languages (178). Chumbow's position is that, for the African languages to be developed to serve their supposed functions, the above problems must be given considerable attention. This is a strong point that must not be neglected if our languages are to forge ahead.

One aspect of the development of African languages which engages Chumbow's attention most, is science and technology. He sees indigenous languages as the foundation for science and technology. According to him, indigenous languages are the vectors of indigenous science, culture and knowledge and should therefore be identified, developed and elaborated (2003:180-181). This implies that for Africa to contribute its quota in science and technology, there is the need to develop a culture of science and technology. This can be achieved through the demystification and democratisation of access to knowledge in science and technology by the use of community languages in education and the delivery of social services for national development. Chumbow therefore, advises that 'African Governments should endeavour to allocate the funds necessary for the enterprise' (2003:190).

This is an advice that must not be neglected because national development in Africa has an intimate connection with language development. The development of Africa is therefore dependent on the development of African languages.

Capo (1990), like Ngugi (2003) and Chumbow (2003), also examines the status of African languages. To him, they have marginal status because they are not the official languages of the countries where they are spoken. They are "unjustifiably discriminated against". He says that "this situation must change so that African languages can be used to democratise knowledge and power" (2). He sees the over use and teaching of European languages as media of instruction throughout the formal school system as one of the reasons for the high degree of drop outs from the school system, and that it is bound to impede economic take-off of our countries and misdirect our priorities (2). He therefore maintains that more proactive steps be taken and a comprehensive scheme be designed to ensure the effective implementation of the language planning agenda for African languages (3).

The solution to the above problem lies in the effective commitment of the linguists to the liberation struggle, such as providing an optimal orthographic system of their languages. The liberation of African languages must follow from their re-acquiring the property of intellectualisation (Capo:4-6). It is demonstratively true from Capo's words that African languages need to be intellectualised as an impetus to their functional survival.

\subsection{Tiv Language Planning}

The Tiv language too does not seem to be an exception from the many African languages that are in dire need of language engineering efforts and programmes. One of the Tiv language scholars, G.A. Gundu (1990) admits the fact that the Tiv language especially "Tivphone literature" as he calls it - the literature written in the Tiv language, has a lot of obstacles which need to be surmounted before the language can forge head. He categorises these obstacles into two; linguistic and sociological. The linguistic ones include (a) inconsistent language policy, (b) dichotomy between the oral and written forms and (c) challenges of the orthography. While the sociological ones cover questions bothering on issues like (a) the threat of the majority languages' literatures over the Tiv language and (b) the issues about distribution (Gundu 1990:146).

He also emphasises that in the Tiv language there is a great gap between the written and spoken modes. "A problem which appears difficult to reconcile despite various recognisable efforts made by several scholars in this direction" (1990:148). According to him, this problem spills over into the transcription. He identifies the disparities in transcription. The first is by indicating tone marks while the second is by ignoring them. These difficulties, according to him, have led to inconsistencies in the transcription of the Tiv language and the orthography. Gundu also addresses the issue of the orthography. He sees the challenges posed by the Tiv orthography of great concern for the survival of Tivphone literature. He maintains that unlike some other Nigerian languages, Tiv language has an orthography published by the National Language Centre (1983) with Robert G. Armstrong as the author but unfortunately, it has not reached the hands of the ordinary users of the language but rather only among linguists.

This problem is quite practical because the existence of the Tiv orthography is not known to many and the most important issue emanating from it is the distance between it and its users. This has generated a lot of inconsistencies in the spelling of Tiv words. He (Gundu) enumerates inconsistencies in the works of such writers like Suemo Chia - AdanWade Kohol Ga (1982) and G.T. Humbe - Tar Homon U Botwev (1961). These issues relating to the orthography must not be taken for granted because they have considerable influence on the development of the Tiv language. 
Gundu (1990) however does not only identify the problem but also suggests some useful steps and strategies for the development of Tiv language. The first strategy he mentions concerns the National Policy on Education (NPE). According to him "if the language aspect of the NPE is implemented meaningfully in the Tiv speaking areas of the country, it will boost the production and consumption of the literature"(153). The second strategy he dwells on is on the gap between the authors and their readers. He states that 'another factor that is likely to motivate and increase readership of Tivphone literature is the conscious efforts made by some of the authors to narrow the gap of interest between them and the readers of their works' (153). Another prospect he identifies "lies in modern technology where visual and phonological gadgets have been developed which can be used for the documentation and preservation of the oral form of the literature without trying to reduce it to writing"(154).

From Gundu's (1990) views, we can deduce that the Tiv language literature has some obstacles militating against its development, but there is hope for its survival if careful and thoughtful planning is done. Though his views are based on Tivphone literature, they are seemingly representative of the state of the Tiv language because the fundamental issues he has raised are not restricted only to the literature but to the entire language.

R.G. Armstrong's (1986) publication, Orthographies of Nigerian Languages: Manual Four, in which the orthography of the Tiv language is contained was a good effort but there seems to be no further development as promised in the NPE. Captain R.C. Abraham's books: A Dictionary of the Tiv Language (1940), The Principles of Tiv (1940), Tiv Reader for European Students (1940), Tiv Phonetic and Tonal Principles (1940), The Bantu Features of Tiv (1940), English-Tiv Dictionary (1940) and The Tiv People (1940) made some enduring impact on the language especially in relation to its structure, phonology and lexico-semantics. What is evident today is a lack of interest and commitment. Abraham is not even a Tiv person. He was a European Administrator in Tivland, but gave this amount of attention to the Tiv language. If only Tiv intellectuals gave this same degree of attention to their language, it could have grown by now to an inestimable level. What this suggests is the need for a renewed sense of commitment to the Tiv language by the Tiv intellectuals.

The above review has revealed that the planning and development of Tiv language like many African languages have suffered some setbacks in many respects and therefore require urgent attention. Also considering the fact that some of the literature on the language seems a bit old, the present study seeks to investigate empirically the current state of the language, and most specifically the functions it currently serves within its speech community and the implications thereof.

\section{Methodology}

\subsection{Research Design}

The survey design is adopted for this study. The survey design focuses on large populations. This research design is adopted for this research because of the large data involved. As a result, not all the subjects or items in the population can be studied. Hence the survey design selects a sample from the population using some techniques of sampling (Asika $2006,13)$. One major feature of all survey designs is lack of control which leads to a high and objective degree of reliability. The focus of this research is on language planning and so the survey design is appropriate for it in the sense that it makes room for representative and objective views about the problem in question.

\subsection{Area of the Study}

This research covers the Tiv speaking area of Benue State. This implies that the data is restricted to the Tiv people located in Benue State and not those in the other neighbouring states like Nassarawa, Taraba, Plateau, Cross-River etc. This restriction is because of space and time factors.

\subsection{Population of the Study}

The population of this study comprises of twenty eight (28) respondents each from the following stakeholders in Tiv language planning: The clergy, second class chiefs, lecturers, teachers, students, linguists, parents, top government functionaries and members of Tiv Language Studies and Development Association. These sum up to two hundred and fifty two (252).

The choice of the respondents is because of their roles as stakeholders/actors in language planning and the Tiv language in particular which implies that they will have more informed views about the subject matter. The choice of twenty eight from each group is for equal distribution based on the number of Tiv Local Government Areas in Benue State. That is, two each in every group from the fourteen (14) local governments.

\subsection{Sample and Sampling}

The total of two hundred and fifty two (252) respondents that represent the population of the study comprises of reverend fathers, pastors, second class chiefs, lecturers in Benue State University, and College of Education KatsinaAla, Primary and Secondary School teachers, Parents, Directors in the Benue State Ministry of Education, State Universal Basic Education Board (SUBEB), Teaching Service Board, Secondary School and University students.

\subsection{Instrumentation and Method of Data Collection}

The instrument used for data collection for this research is the questionnaire (Appendix 1). The items developed in the questionnaire relate directly to the planning of Tiv language function(s). The questionnaire has three possible options to show acceptance, rejection or indifference as can be seen below 


$\begin{array}{ll}{[\mathrm{Y}]} & \text { YES } \\ {[\mathrm{N}]} & \text { NO } \\ {[\mathrm{NS}]} & \text { NOT SURE }\end{array}$

This is to enable the respondents to indicate the responses that suit their opinion. The 11-item questionnaire was administered to the target respondents (personally) with an appeal to respond to the questions appropriately, honestly and objectively.

\subsection{Validation of the Instrument}

The developed instrument - the questionnaire - was validated so as to ensure reliability. The items in the questionnaire went through a thorough screening exercise by experts/linguists in the department of languages and linguistics, Benue State University, Makurdi. The criteria for inclusion of items in the questionnaire include simplicity, clarity, appropriateness of word use and relevance to the research questions.

\subsection{Method of Data Presentation and Analysis}

The completed questionnaires were collected and presented in tables using a percentile analytical method. The analysis is done using the formula below

$$
\frac{N R}{T N R} X \frac{100}{1}=\%
$$

$\mathrm{NR}=$ Number of Respondents

TNR $=$ Total Number of Respondent

$\%=$ Percentages of Population Sampled.

\section{Data Analysis and Discussion of Findings}

Below is the analysis of the data obtained from two hundred and twenty eight (228) respondents recovered out of the two hundred and fifty two (252) that were administered. The analysis is in two parts. The first is the tabular representation and analysis and the second is the discussion of findings.

\subsection{Data Presentation and Analysis}

Question 1. Is there enough written literature in Tiv language to enable it function as a national and or regional language?

Table 1. Respondents' views on the availability and adequacy of Tiv written literature

\begin{tabular}{lcl}
\hline RESPONSES & NUMBER OF RESPONDENTS & PERCENTAGES (\%) \\
\hline YES & 90 & $39.47 \%$ \\
NO & 122 & $53.51 \%$ \\
NOT SURE & 16 & $07.02 \%$ \\
TOTAL & 228 & $100 \%$ \\
\hline
\end{tabular}

From table 1, we can see that 90 respondents agreed that there is enough written literature in Tiv, 122 disagreed and 16 were not sure. This suggests that the existing written literature in Tiv language is not adequate enough.

Question 2. Is Tiv Language adequate enough to be used in mathematical processes?

Table 2. Respondents' views on the adequacy of Tiv language for mathematical processes

\begin{tabular}{lcl}
\hline RESPONSES & NUMBER OF RESPONDENTS & PERCENTAGES (\%) \\
\hline YES & 40 & $17.53 \%$ \\
NO & 164 & $71.93 \%$ \\
NOT SURE & 24 & $10.53 \%$ \\
TOTAL & 228 & $100 \%$ \\
\hline
\end{tabular}

As table 2 shows, 40 respondents agreed that Tiv language is adequate enough to be used in mathematical processes, 164 disagreed while 24 were not sure. Since the number of respondents who disagreed are more than those who agreed and or were not sure, it is indicative that Tiv language is not adequate enough to be used in mathematical and by extension scientific and technological processes. 
Question 3a Is Tiv Language taught as a subject in schools in the Tiv speaking areas of Benue State?

Table 3a. Respondents' views on the teaching of Tiv language as a school subject in Benue State

\begin{tabular}{lcl}
\hline RESPONSES & NUMBER OF RESPONDENTS & PERCENTAGES (\%) \\
\hline YES & 16 & $07.02 \%$ \\
NO & 186 & $81.58 \%$ \\
NOT SURE & 26 & $11.40 \%$ \\
TOTAL & 228 & $100 \%$ \\
\hline
\end{tabular}

Table $3 \mathrm{a}$ above shows that shows that 16 respondents agreed that Tiv language is taught as a subject in schools in the Tiv speaking areas of Benue State, 186 disagreed while 26 were not sure. This is suggestive that Tiv language is not taught as a subject in schools even in the Tiv speaking areas of Benue State.

Question 3b If "Yes" to "a" above, is there any commitment by teachers to its teaching?

Table 3b. Respondents' views on the commitment to the teaching of Tiv language in Benue state

\begin{tabular}{lcl}
\hline RESPONSES & NUMBER OF RESPONDENTS & PERCENTAGES (\%) \\
\hline YES & 0 & $0.0 \%$ \\
NO & 16 & $100 \%$ \\
NOT SURE & 0 & $0.0 \%$ \\
TOTAL & 16 & $100 \%$
\end{tabular}

The responses for question $3 \mathrm{~b}$ as presented in table $3 \mathrm{~b}$ show that all the respondents disagreed that there is no commitment by teachers to the teaching of Tiv language in the Tiv speaking areas of Benue State. No respondents agreed nor were not sure. This therefore indicates that even in the instance where little efforts are made to teach Tiv language, there is no commitment to it.

Question 4. Are there enough Tiv language teachers?

Table 4. Respondents' views on the availability and adequacy of Tiv language teachers

\begin{tabular}{lcl}
\hline RESPONSES & NUMBER OF RESPONDENTS & PERCENTAGE (\%) \\
\hline YES & 18 & $07.89 \%$ \\
NO & 202 & $88.60 \%$ \\
NOT SURE & 08 & $03.51 \%$ \\
TOTAL & 228 & $100 \%$ \\
\hline
\end{tabular}

18 respondents, as can be seen in table 4, agreed that there are enough Tiv language teachers, 202 disagreed while 08 were not sure. This suggests a dearth of Tiv language teachers.

Question 5. Is Tiv Language used as a medium of instruction in the primary and junior secondary schools in the Tiv speaking areas of Benue State?

Table 5. Respondents' views on the use of Tiv language as a medium of instruction in schools in Benue State

\begin{tabular}{lcl}
\hline RESPONSES & NUMBER OF RESPONDENTS & PERCENTAGE (\%) \\
\hline YES & 24 & $10.53 \%$ \\
NO & 178 & $78.07 \%$ \\
NOT SURE & 26 & $11.40 \%$ \\
TOTAL & 228 & $100 \%$ \\
\hline
\end{tabular}

Table 5 above shows that 24 respondents agreed that Tiv language is used as the medium of instruction in the primary and junior secondary schools in the Tiv speaking areas of Benue State, 178 disagreed, while 26 were not sure. This indicates that Tiv language is not used as a medium of instruction in the primary and junior secondary schools in Benue State, even though the NPE stipulates so. 
Question 6.Should Tiv language be made a compulsory school subject for all Tiv children in the primary and secondary schools in Benue State?

Table 6. Respondents' views on making Tiv language a compulsory school subject in the primary and secondary schools in Benue State

\begin{tabular}{lcl}
\hline RESPONSES & NUMBER OF RESPONDENTS & PERCENTAGE (\%) \\
\hline YES & 208 & $91.23 \%$ \\
NO & 06 & $02.63 \%$ \\
NOT SURE & 14 & $06.14 \%$ \\
TOTAL & 228 & $100 \%$ \\
\hline
\end{tabular}

Table 6 above indicates that 208 respondents agreed that there is need to make Tiv language a compulsory subject for all Tiv children in the primary and secondary schools, 06 disagreed, while 14 were not sure. This indicates that there is need to make Tiv language a compulsory school subject for Tiv children in the primary and secondary schools in Benue State.

Question 7. Is there any need for a B.A. degree in Tiv Language?

Table 7. Respondents' views on the need for a B.A. degree in Tiv language

\begin{tabular}{lcl}
\hline RESPONSES & NUMBER OF RESPONDENTS & PERCENTAGE (\%) \\
\hline YES & 216 & $94.34 \%$ \\
NO & 04 & $01.75 \%$ \\
NOT SURE & 08 & $03.51 \%$ \\
TOTAL & 228 & $100 \%$ \\
\hline
\end{tabular}

In table 7 above, we can see that 216 respondents agreed that there is need for a B.A. degree in Tiv language, 04 disagreed, while 08 were not sure. This suggests a genuine need for a B.A. degree in Tiv Language in Nigerian Universities in order to train specialists in the language.

Question 8. Do educated Tiv parents speak with their children in Tiv language in the homes?

Table 8. Respondents' views on educated parents' use of Tiv language in the homes with their children

\begin{tabular}{lcl}
\hline RESPONSES & NUMBER OF RESPONDENTS & PERCENTAGE (\%) \\
\hline YES & 08 & $03.51 \%$ \\
NO & 218 & $95.61 \%$ \\
NOT SURE & 02 & $00.88 \%$ \\
TOTAL & 228 & $100 \%$ \\
\hline
\end{tabular}

From Table 8 above, 08 respondents agreed that educated Tiv parents speak Tiv language to their children at home, 218 disagreed while 02 was not sure. This shows that most educated Tiv parents do not speak Tiv language to their children at home.

Question 9. Does Tiv Language feature frequently on Radio in Benue State?

Table 9. Respondents' views on the frequency of Tiv language on radio in Benue State

\begin{tabular}{lcl}
\hline RESPONSES & NUMBER OF RESPONDENTS & PERCENTAGE (\%) \\
\hline YES & 64 & $28.07 \%$ \\
NO & 146 & $64.04 \%$ \\
NOT SURE & 18 & $07.89 \%$ \\
TOTAL & 228 & $100 \%$ \\
\hline
\end{tabular}


Table 9 above shows that 64 respondents agreed that Tiv Language features frequently on Radio in Benue State, 146 disagreed while 18 were not sure. Based on the data above, we can deduce that Tiv language does not feature prominently and frequently on radio in Benue State.

Question 10. Does Tiv Language feature frequently on Television in Benue State?

Table 10. Respondents' views on the frequency of Tiv language on television in Benue State

\begin{tabular}{lcl}
\hline RESPONSES & NUMBER OF RESPONDENTS & PERCENTAGE (\%) \\
\hline YES & 04 & $01.75 \%$ \\
NO & 218 & $95.61 \%$ \\
NOT SURE & 06 & $02.63 \%$ \\
TOTAL & 228 & $100 \%$ \\
\hline
\end{tabular}

04 respondents as can be seen in table 10 above agreed that Tiv Language features frequently on Television in Benue State, 218 disagreed, while 06 were not sure, suggesting that Tiv Language features very infrequently on television in Benue State.

Question 11. Does Tiv Language feature frequently in the Print Media?

Table 11. Respondents' views on the frequency of Tiv language in the print media in Benue State

\begin{tabular}{lcl}
\hline RESPONSES & NUMBER OF RESPONDENTS & PERCENTAGE (\%) \\
\hline YES & 02 & $00.88 \%$ \\
NO & 222 & $97.37 \%$ \\
NOT SURE & 04 & $01.75 \%$ \\
TOTAL & 228 & $100 \%$ \\
\hline
\end{tabular}

Table 11 above shows that 02 respondent agreed that Tiv Language features frequently in the print media in Benue State, 222 disagreed, while 04 were not sure. Since those who disagreed are divergently higher, it is indicative that Tiv Language does not feature frequently in the print media in Benue State.

\subsection{Discussion of Findings}

The data analysis has answered a lot of fundamental questions regarding the functional aspect of Tiv language planning and development. The functions of the language that were investigated in this study include, use in schools as a medium of structure, as a school subject as well as a degree course, the media, science and technology and the home, among others. The analysis suggests a bleak status as most of the questions were answered in the negative.

For example the pedagogical aspect of Tiv language which is crucial to the survival of any language does not seem to receive the desired attention. There is therefore the need for more to be done by enforcing the teaching of Tiv language in schools. As part of such efforts, a B.A. degree in Tiv language becomes one of the necessary and significant prerequisites. The Federal and State Governments' commitment which, as the data has proven, is lacking has to be reaffirmed so as to give the language a firm backing and to make laws and policies in favour of Tiv language.

The positive attitude of parents to Tiv language, especially the need to speak Tiv language to their children, will also be very significant to Tiv language development. The use of Tiv in the media also suffers a dearth of frequency. Tiv native speakers must cultivate the habit of using Tiv language in the media: newspapers, magazines, journals, radio and television. The ministries of education, information, communication and culture need to act positively in this direction.

The findings of this study corroborate the findings of other scholars like Emenanjo (1990), Essien (1990), Williamson (1990) and Jibril (2006) who have identified and decried a similar dearth of attention given to the Nigerian languages. Emenanjo (1990) identifies the need for language engineering efforts to be expedited to develop Nigerian languages. He calls for the establishment of language planning agencies for all Nigerian languages in order to pay more enduring attention to language planning programmes, processes and issues concerning them thereby averting the danger or risk of extinction they stand.

Essien's (1990) views are in many respects similar to Emenanjo's. Acording to him, the status of most Nigerian languages is one of low priority and underdevelopment. He identifies four factors as being responsible for the current state of Nigerian languages. These include: the Constitution, the National Policy on Education, the non-implementation of policy and the attitude of the Nigerian elite. The attitude of educated parents towards Tiv language as the findings of the present study have shown goes a long way to confirm Essien's views.

Williamson (1990) like Emenanjo and Essien sees the cooperation between the speakers of the languages and the language planning agencies as a useful strategy for the planning development of Nigerian languages. To her, the role of the speakers of the language is key to its development and survival. 
Jibril (2006) also reiterates the same position as Emenanjo as he laments the danger of extinction hanging on Nigerian languages and therefore calls for some significant efforts to be made to avert this ugly development.

Tiv language, like many other Nigerian languages, belongs to this category of endangered languages as the findings have shown that it has a week functional status.

\section{Conclusion}

The findings of this study have shown that the current functional status of Tiv language is weak as it does not seem to be serving the functions it is supposed. Therefore some conscious efforts need to be made to develop and enable it function optimally. Such efforts, as seen in the analysis and as contained in the recommendations, must not be taken for granted so as to ensure the survival, growth and development of the language. The effective application of such strategies like the writing of books for Tiv language, encouragement of authors, parents' predilections for speaking Tiv language to their children, the promotion of Tiv language in the schools and the media, language sensitisation, revival and promotion, Government's commitment and the linguists' involvement and commitment will go a long way in the development of Tiv language and culture.

But above all, the role and commitment of the native speakers who are the owners of the language are the most crucial factors in the development of Tiv language. As Williamson (1990:144) maintains, the task of developing indigenous languages is the principal responsibility of the owners of the language. If they care for them, they will develop them, if they do not, then the language will inevitably die in favour of another language. Tiv language too if not given the desired attention will gradually die in favour of English language, which is already engulfing it as cataract does to the lens of the eye. The Tiv native speakers must therefore rise up to this challenge in order to do to their language what other intellectuals in history have done to theirs (Ngugi 2003).

\section{Recommendations}

Based on the results of the study, the following recommendations are made:

i. Tiv Language-medium authors be encouraged through research grants and sponsorship of their publications.

ii. Tiv language be made a compulsory subject in schools in Benue State especially among Tiv pupils and students. In addition, the medium of instruction in the primary and junior secondary schools in the Tiv speaking areas of Benue State be Tiv language.

iii. A B.A. degree in Tiv language be mounted in Nigerian Universities.

iv. An academy be established for Tiv language.

v. Tiv parents, especially educated parents, should endeavour to speak to their children in Tiv language.

vi. $\quad$ More teachers be trained for Tiv language.

vii. Tiv-medium newspapers and media houses be established.

viii. The number of radio and especially television hours for Tiv language in Benue State be increased considerably.

ix. Language sensitisation efforts and cultural revival movements for Tiv language be embarked upon.

x. The Tiv native speakers should show commitment, and form positive attitudes towards their language. This is cardinal to Tiv language development because the bulk of the task of developing Tiv language rests in their hands.

\section{Suggestions for Further Study}

This study embarked on the ambitious task of investigating the planning of Tiv language but focused on only one aspect of it: status planning. The other two: corpus and acquisition were not dealt with. Further research in these directions would prove useful in providing more holistic and illuminating insights about the language.

Even within the status planning, not all the functions of the language were investigated such as the religious and administrative ones which are equally crucial in status planning. More in-depth studies addressing all the key functions of the language be embarked upon.

The method adopted for this study was quantitative using the instrument of the questionnaire which certainly has its limitations. Studies utilising other approaches like qualitative or mixed methods would bring complementary insights to bear on an objective understanding of the subject matter.

\section{References}

Abraham, R.C. (1940). The Tiv people (2nd ed).. London: Crown Agents.

--- (1940). A dictionary of the Tiv language. London: Crown Agents.

--- (1940). The principles of Tiv. London: Crown Agents.

--- (1940). Tiv reader for European students. London: Crown Agents.

--- (1940). Tiv phonetic and tonal principles. London: Crown Agents.

--- (1940). The Bantu features of Tiv. London: Crown Agents. 
--- (1940). English-Tiv dictionary. London: Crown Agents.

Armstrong, R.G. (Ed.). (1986). Orthographies of Nigerian languages: Manual IV. National Language Centre, Federal Ministry of Education, Lagos.

Appel R. and Myusken, P. (1990). Language contact and bilingualism. London: Edward Arnold.

Asika, N. (2006). Research methodology in the behavioural sciences. Lagos: Longman.

Benji, W. (1989). Swahili and the Bantu Languages. In Comrie, B (Ed.), The Major World Languages. London: Routledge. 991.

Blench, R.M. (2002). Research on minority languages of Nigeria in 2001. Ogmios

Capo, HBC. (1990). Comparative linguistics and language engineering in Africa. In Emenanjo, E.N (Ed.), Multilingualism, Minority Languages and Language Policy in Nigeria. Agbor: Central. 1-9.

Chia, S. (1982). Adan-Wade kohol ga. Makurdi: Satos.

Chumbow, B.S. (2003). The Language Question and National Development in Africa. In Mkamdawire, T. (Ed.), African Intellectuals. CODESRIA.

Cooper, Robert. (1989). Language planning and social change. Cambridge: Cambridge UP.

Crozier, D. \& Blench, R. (1992). An index of Nigerian languages (2nd Ed.). Dallas: SIL.

Emenanjo, E.N. (1990). In the tradition of the majors: Lessons in language engineering for the minority languages. In Emenanjo, E.N (Ed.), Multilingualism, Minority Languages and Language Policy in Nigeria. Agbor: Central. 88-98.

Essien, O.E. (1990). The future of minority languages. In Emenanjo, E.N (Ed.), Multilingualism, Minority Languages and Language Policy in Nigeria. Agbor: Central.155-168.

Ethnologue. (2015). Listing of Nigerian Languages. http://www.ethnologue.com/country/NG

Federal Government of Nigeria. (2004) National policy on education (4 ${ }^{\text {th }}$ Ed). Lagos: Federal Government Press.

Ferguson, C. (1968). Language development. In Fishman, J. A et al. (Eds.), Language Problems of Developing Nations. New York: John Wiley and Sons. 27-35.

Ferguson, C. (1983). Language planning and language change. In Cobarrubias, J and Fishman, J.A. Fishman (Eds.), Progress in Language Planning: International Perspectives. Berlin: Mouton. 29-40.

--- (1974). Language Modernisation and planning in comparison with other types of national modernisation and planning. In Fishman, J.A. (Ed.), Advances in Language Planning The Hague: Mouton. 79-102.

Gbor, W.T. (1986). Study Tiv language._Zaria: Gaskiya.

Gorman, T. P. (1973). Language allocation and language planning in a developing nation. In Rubin, J. and Shuy, R. (Eds.). Language Planning: Current Issues and Research. Washington: Georgetown Up. 72-82.

Gundu, G.A. (1990). Problems and prospects of minority language literatures: The case of Tivphone literature. In Emenanjo, E.N (Ed.), Multilingualism, Minority Languages and Language Policy in Nigeria. Agbor: Central. 145-154

Gyanggyang, S. (Ed.). (2000). History of the NKST church: 1911-2000. Makurdi: Lamp and Word.

Haugen, E. (1983). The implementation of corpus: Theory and practice. In Cobarrubias, J. and Fishman, J.A. Fishman (Eds.), Progress in Language Planning: International Perspectives. Berlin: Mouton. 269-289.

Haugen, E. (1971). Instrumentalism in language planning. In Rubin, J. and Jernudd, B.J. (Eds.), Can Languages be Planned? Sociolinguistic Theory and Practice for Developing Nations. Honolulu: The University Press of Hawaii. 281289.

Hembe, G.N. (2005). J.S. Tarka: The dilemma of ethnic minority politics in Nigeria. Makurdi: Aboki.

Humbe, G.T. (1961). Tar homon u Botwer. Zaria: Gaskiya.

Jernudd, B.H. and Das Gupta, J. (1971). Towards a Theory of Language Planning. In Rubin, J. and Jernudd, B.J. (Eds.), Can Languages be Planned? Sociolinguistic Theory and Practice for Developing Nations. The Hague: Mouton. 103124.

Jibril, M. (2006). The future of Nigerian languages. International Journal of Linguistics and Communication. 1. 1-4

Kloss, H. (1969). Abstand languages and ausbau languages. Anthropological Linguistics. 9(7). 29-41.

Lucas, C. and Nercissians, E. (1988). A game - theoretical approach to language planning. In Jorgensen, J.N et al (Eds.), Bilingualism in Society and School. Philandelphia: Multilingual matters. 1-10.

Makar, T. (1994). The history of political change among the Tiv in the $19^{\text {th }}$ and $20^{\text {th }}$ centuries. Enugu: Fourth Dimension.

National Population Commission. Census 2006 Result.

Rubin, J. (1971). Evaluation and language planning. In Rubin, J. and Jernudd, B.J. (Eds.), Can Languages be Planned? Sociolinguistic Theory and Practice for Developing Nations. Honolulu: The University Pres of Hawaii. 217-252.

Sai, Ak. Akiga's story. (1939). East, R. (Trans.). London: Oxford Up. 
Stewart, W. (1968). A sociolinguistic typology for describing national multilingualism. In Fishman, J.A. (Ed.). Readings in the Sociology of Language. The Hague: Mouton. 531-545.

Tauli, V. (1968). Introduction to a theory of language planning. Uppsala: Almqvist and Wiksell.

Wa Thiongo, N. (2003). Europhone or African memory: The challenges of the pan-Africanist intellectual in the era of globalisation. In Mkamdawire, T. (Ed.), African Intellectuals. CODESRIA.

Williamson, K. (1990). Development of minority languages: Publishing problems and prospects. In Emenanjo, E.N (Ed.), Multilingualism, Minority Languages and Language Policy in Nigeria. Agbor: Central. 118-144.

Yuhe, D.V. (1978). The encounter of Tiv religious and moral values with Catholicism in the time of secularisation (Unpublished Ph.D. Thesis). Pontificia Studicrum Universities at Thomas Aquinate in Urbe, Rome.

\section{Appendix}

\section{Sample Questionnaire}

Instructions:

Tick $[\sqrt{ }]$ Y for YES, N for NO, and NS for NOT SURE as appropriate, from the alternatives provided in each of the questions below.

1. Is there enough written literature in Tiv language to enable it function as a national and or regional language?

$[\mathrm{Y}][\mathrm{B}]$

$[\mathrm{N}][\mathrm{B}]$

[NS][ ]

2. Is Tiv language adequate enough to be used in mathematical process?

$[\mathrm{Y}][\mathrm{]}]$

$[\mathrm{N}][\mathrm{B}]$

[NS][ ]

3a. Is Tiv language taught as a subject in schools in the Tiv speaking areas of Benue State?

[Y][ ]

$[\mathrm{N}][\mathrm{C}]$

[NS][ ]

3b. If Yes to 13 above, is there any commitment by teachers to its teaching?

$[\mathrm{Y}][\mathrm{C}]$

$[\mathrm{N}][\mathrm{C}]$

[NS][ ]

4. Are there enough Tiv Language Teachers?

$[\mathrm{Y}][\mathrm{B}]$

$[\mathrm{N}][\mathrm{H}]$

[NS][ ]

5. Is Tiv language used as a medium of instruction in the primary and junior secondary schools in the Tiv speaking areas of Benue State?

$[\mathrm{Y}][\mathrm{]}]$

$[\mathrm{N}][\mathrm{B}]$

[NS][ ]

6. Should Tiv language be made a compulsory school subject for all Tiv children in the primary and secondary schools in Benue State?

$[\mathrm{Y}][\mathrm{B}]$

$[\mathrm{N}][\mathrm{H}]$

[NS][ ]

7. Is there any need for a B.A. Degree in Tiv Language?

[Y][ ]

[N][ ]

[NS][ ] 
8. Do educated Tiv parents speak with their children in Tiv language in the homes?

[Y][ ]

[N][ ]

[NS][ ]

9. Does Tiv language feature frequently on Radio in Benue State?

[Y][ ]

[N][ ]

[NS][ ]

10. Does Tiv language feature frequently on Television in Benue State?

[Y][ ]

[N][ ]

[NS][ ]

11. Does Tiv language feature frequently in the print media?

[Y][ ]

[N][ ]

[NS][ ] 